Proyecciones Journal of Mathematics

Vol. 37, $\mathrm{N}^{\circ}$ 4, pp. 627-635, December 2018.

Universidad Católica del Norte

Antofagasta - Chile

\title{
Fekete-Szego problem for certain analytic functions defined by $q$-derivative operator with respect to symmetric and conjugate points
}

\author{
S. O. Olatunji \\ Federal University of Technology, Nigeria \\ and \\ H. Dutta \\ Gauhati University, India \\ Received : January 2018. Accepted : April 2018
}

\begin{abstract}
Recently, the q-derivative operator has been used to investigate several subclasses of analytic functions in different ways with different perspectives by many researchers and their interesting results are too voluminous to discuss. For example, the extension of the theory of univalent functions can be used to describe the theory of q-calculus, $q$-calculus operator are also used to construct several subclasses of analytic functions and so on. In this work, we considered the FeketeSzego problem for certain analytic functions defined by $q$-derivative operator with respect to symmetric and conjugate points. The early few coefficient bounds were obtained to derive our results.
\end{abstract}

AMS Subject Classification : 30C45, 30C50

Keywords : Analytic function, univalent function, $q$-derivative operator, subordination, coefficient bounds, coefficient inequalities. 


\section{Introduction}

Let $A$ represent the class of analytic functions $f$ defined by the unit disc $U=\{z:|z|<1\}$ and given by

$$
f(z)=z+\sum_{n=2}^{\infty} a_{n} z^{n} .
$$

Also, let $S \subset A$ consisting of functions which are univalent inside $U$. The well-known classes of analytic functions are starlike and convex functions which their geometrical condition satisfies $\operatorname{Re}\left(\frac{z f^{\prime}(z)}{f(z)}\right)>0$ and $\operatorname{Re}\left(1+\frac{z f^{\prime \prime}(z)}{f^{\prime}(z)}\right)>0$.

Let $S_{s}^{*}$ be the subclass of $S$ consisting of functions given by (1.1) satisfying the condition

$$
\operatorname{Re}\left(\frac{z f^{\prime}(z)}{f(z)-f(-z)}\right)>0 \quad z \in U .
$$

This class of functions referred to the class of starlike function with respect to symmetric points.

Let $S_{c}^{*}$ be the subclass of $S$ consisting of functions given by (1.1) satisfying the condition

$$
\operatorname{Re}\left(\frac{z f^{\prime}(z)}{f(z)-\overline{f(\bar{z})}}\right)>0 \quad z \in U .
$$

This class of functions referred to the class of starlike function with respect to conjugate points.

Let $S_{s}^{c}$ be the subclass of $S$ consisting of functions given by (1.1) satisfying the condition

$$
\operatorname{Re}\left(\frac{\left(z f^{\prime}(z)\right)^{\prime}}{(f(z)-f(-z))^{\prime}}\right)>0 \quad z \in U
$$

This class of functions referred to the class of convex function with respect to symmetric points. 
Let $S_{c}^{c}$ be the subclass of $S$ consisting of functions given by (1.1) satisfying the condition

$$
\operatorname{Re} \frac{\left(z f^{\prime}(z)\right)^{\prime}}{(f(z)-\overline{f(\bar{z})})^{\prime}}>0 \quad z \in U .
$$

This class of functions referred to the class of convex function with respect to conjugate points.

Two analytic functions are said to be subordinate to each other written as $f \prec g$, if there exists a Schwartz function $\omega$ which is analytic in $U$ with $\omega(0)=0$ and $|\omega(z)|<1$, for all $z \in U$, such that $f(z)=g(\omega(z))$, and $f(U) \subset g(U)$.

Goel and Mehrok [4] introduced a subclass of $S_{s}^{*}$ denoted by $S_{s}^{*}(A, B)$ in terms of subordination which their geometrical condition satisfies

$$
\operatorname{Re} \frac{2 z f^{\prime}(z)}{f(z)-f(-z)} \prec \frac{1+A z}{1+B z} \quad-1 \leq B<A \leq 1 \quad z \in U .
$$

For a subclass of $S_{c}^{*}$ denoted by $S_{c}^{*}(A, B)$ in terms of subordination and their geometrical condition satisfies

$$
\operatorname{Re} \frac{2 z f^{\prime}(z)}{f(z)-\overline{f(\bar{z})}} \prec \frac{1+A z}{1+B z} \quad-1 \leq B<A \leq 1 \quad z \in U .
$$

Also, a subclass of $S_{s}^{c}$ denoted by $S_{s}^{c}(A, B)$ in terms of subordination and their geometrical condition satisfies

$$
\operatorname{Re} \frac{2\left(z f^{\prime}(z)\right)^{\prime}}{(f(z)-f(-z))^{\prime}} \prec \frac{1+A z}{1+B z} \quad-1 \leq B<A \leq 1 \quad z \in U .
$$

Lastly, a subclass of $S_{c}^{c}$ denoted by $S_{c}^{c}(A, B)$ in terms of subordination and their geometrical condition satisfies

$$
\operatorname{Re} \frac{2\left(z f^{\prime}(z)\right)^{\prime}}{(f(z)-\overline{f(\bar{z})})^{\prime}} \prec \frac{1+A z}{1+B z} \quad-1 \leq B<A \leq 1 \quad z \in U .
$$

See details in [3], [4] and [14].

For $q \in(0,1)$, Jackson's $q$-derivative [[6],[7]] of a function $f \in A$ is given by

$$
\left(D_{q} f\right)(z)= \begin{cases}\frac{f(z)-f(q z)}{(1-q) z}, & \text { for } z \neq 0 \\ f^{\prime}(0), & \text { for } z=0\end{cases}
$$


where $D_{q}^{2} f(z)=D_{q}\left(D_{q} f(z)\right)$. If follows from 1a that

$$
\left(D_{q} f\right)(z)=1+\sum_{n=1}^{\infty}[n]_{q} a_{n} z^{n-1}, \text { where } \quad[n]_{q}=\frac{1-q^{n}}{1-q}
$$

and it is called the basic number $n$. If $q \longrightarrow 1^{-}$then $[n]_{q} \longrightarrow n$. See details [2], [11], [12] and [13].

Definition A : Let $P$ denote the class of Caratheodory functions with positive real part in $U$. Also, let $P\left(p_{k}\right)$ where $k \in[0, \infty)$ denote the family of functions $P$, such that $p \in P$ and $p \prec p_{k}$ in $U$, and the function $p_{k}$ maps the unit disk conformally onto the region $\Omega_{k}$ such that $1 \in \Omega_{k}$ and

$$
\partial \Omega_{k}=\left\{u+i v: u=k \sqrt{(u-1)^{2}+v^{2}}\right\} .
$$

It is noted that, the domain $\Omega_{k}$ is elliptic for $k>1$, parabola for $k=1$ and hyperbola when $k \in(0,1)$.

Kanas and Wisniowska [9] and [10] shown that the extremal functions $p_{k}(z)$ for conic region are given below:

For $k=0$, we have

$$
p_{0}(z)=\frac{1+z}{1-z}=1+2 z+2 z^{2}+2 z^{3}+2 z^{4}+\ldots
$$

For $k=1$

$$
p_{1}(z)=1+\frac{2}{\pi} \log ^{2}\left(\frac{1+\sqrt{z}}{1-\sqrt{z}}\right)=1+\frac{8}{\pi^{2}} z+\frac{16}{3 \pi^{2}} z^{2}+\frac{184}{45 \pi^{2}} z^{3}
$$

and lastly for $k \in(0,1)$ and $A=A(k)=\frac{2}{\pi} \arccos k$ we obtain $\mathrm{p}_{k}(z)=$ $1+\frac{2}{1-k^{2}} \sinh ^{2}(A(k) \operatorname{arctanh} \sqrt{z})=\frac{1}{1-k^{2}} \cos \left\{A(k) i \log \frac{1+\sqrt{z}}{1-\sqrt{z}}-\frac{k^{2}}{1-k^{2}}\right\}$

$$
1+\frac{2 A^{2}}{1-k^{2}} z+\frac{4 A^{2}+2 A^{4}}{3\left(1-k^{2}\right)} z^{2}+\frac{\frac{46 A^{2}}{15}+\frac{8 A^{4}}{3}+\frac{4 A^{6}}{15}}{3\left(1-k^{2}\right)} z^{3}+\ldots
$$

and for $k>1$ and $u(z)=\frac{z-\sqrt{k}}{1-\sqrt{k z}}$ we have

$$
p_{k}(z)=\frac{1}{k^{2}-1} \sin \left(\frac{\pi}{2 K(k)} \int_{0}^{\frac{u(z)}{\sqrt{k}}} \frac{d t}{\sqrt{1-t^{2}} \sqrt{1-k^{2} t^{2}}}\right)
$$




$$
=1+\frac{\pi^{2}}{4 \sqrt{k\left(k^{2}-1\right) K^{2}(k)(1+k)}}\left\{z+\frac{4 K^{2}(k)\left(k^{2}+6 k+1\right)-\pi^{2}}{4 \sqrt{k\left(k^{2}-1\right) K^{2}(k)(1+k)}} z^{2}+\ldots\right\}
$$

where $K(k)$ indicates the Legendre's complete elliptic integral of the first kind and $K^{\prime}(k)$ is the complementary integrand of $K(k)$ with $k \in(0,1)$ is chosen such that $k=\cosh \left[\left(\pi K^{\prime}(k) / 4 K(k)\right)\right]$. By virtue of

$p(z)=\frac{z f^{\prime}(z)}{f(z)} \prec p_{k}(z)$ or $p(z)=1+\frac{z f^{\prime \prime}(z)}{f^{\prime}(z)} \prec p_{k}(z)$

and the properties of the domains, we have

$$
R(p(z))>R\left(p_{k}(z)\right)>\frac{k}{k+1} .
$$

Motivated by earlier work done by [1], [3], [4], [5], [8] and [14]. In this present investigation, the Fekete-Szego problem for certain classes of analytic function defined by $q$-derivative operator with respect to symmetric and conjugate points were considered. The first few coefficients bound for the classes were obtained which serve as a tools for our results.

Sequel to the next section, the following Lemma and definitions shall be necessary.

Lemma 1.1. [8] Let $\omega(z)=\omega_{1} z+\omega^{2} z^{2}+\ldots \in U$ be so that $|\omega(z)|<1$ in $U$. If $t$ is a complex number then

$$
\left|\omega^{2}+t \omega_{1}^{2}\right| \leq \max \{1,|t|\}
$$

The inequality is sharp for the functions $\omega(z)=z$ or $\omega(z)=z^{2}$.

We shall employ [4] to give the following definition.

Definition 1.1. For the real numbers $k \in[0, \infty), q \in(0,1)$ and for $p_{k}(z)$ as in Definition $A$, we way that a function $f \in A$ is in the class $S_{s}^{*}\left(p_{k}\right)$ if

$$
\frac{2 z\left(D_{q} f(z)\right)}{f(z)-f(-z)} \prec p_{k}(z) .
$$

Definition 1.2. For the real numbers $k \in[0, \infty), q \in(0,1)$ and for $p_{k}(z)$ as in Definition $A$, we say that a function $f \in A$ is in the class $S_{c}^{*}\left(p_{k}\right)$ if

$$
\frac{2 z\left(D_{q} f(z)\right)}{f(z)+\overline{f(\bar{z})}} \prec p_{k}(z) \text {. }
$$


Definition 1.3. For the real numbers $k \in[0, \infty), q \in(0,1)$ and for $p_{k}(z)$ as in Definition $A$, we say that a function $f \in A$ is in the class $S_{s}^{c}\left(p_{k}\right)$ if

$$
\frac{2\left(z\left(D_{q} f(z)\right)\right)^{\prime}}{(f(z)-f(-z))^{\prime}} \prec p_{k}(z) .
$$

Definition 1.4. For the real numbers $k \in[0, \infty), q \in(0,1)$ and for $p_{k}(z)$ as in Definition $A$, we say that a function $f \in A$ is in the class $S_{c}^{c}\left(p_{k}\right)$ if

$$
\frac{2\left(z\left(D_{q} f(z)\right)\right)^{\prime}}{(f(z)+\overline{f(\bar{z})})^{\prime}} \prec p_{k}(z) .
$$

\section{Main Result}

Theorem 2.1. Let $k \in[0, \infty), q \in(0,1)$ and let $p_{k}(z)=1+p_{1} z+p_{2} z^{2}+\ldots$ be defined as in the Definition $A$. If $f$ given by (1.1) belongs to $S_{s}^{*}\left(p_{k}\right)$ then we have

$$
\left|a_{3}-\mu a_{2}^{2}\right| \leq \frac{p_{1}}{[3]_{q}-1} \max \left\{1,\left|\frac{p_{2}}{p_{1}}-\frac{\mu p_{1}\left([3]_{q}-1\right)}{[2]_{q}^{2}}\right|\right\}
$$

(2.1) holds for any complex number $\mu$.

Proof. If $f \in S_{s}^{*}\left(p_{k}\right)$, then there is a Schwarz function $\omega=\omega_{1} z+\omega_{2} z^{2}+$ $\ldots \in U$ such that

$$
\frac{2 z\left(D_{q} f(z)\right)}{f(z)-f(-z)}=p_{k}(\omega(z))
$$

We note that

$$
2 z\left(D_{q} f(z)\right)=(f(z)-f(-z)) p_{k}(\omega(z))
$$

and

$p_{k}(\omega(z))=1+p_{1} \omega_{1} z+\left(p_{1} \omega_{2}+p_{2} \omega_{1}^{2}\right) z^{2}+\left(p_{1} \omega_{3}+2 p_{2} \omega_{1} \omega_{2}+p_{3} \omega_{1}^{3}\right) z^{3}+\ldots$

Applying (2.2), (2.3) and (2.4), we obtain

$$
a_{2}=\frac{p_{1} \omega_{1}}{[2]_{q}}
$$


Fekete-Szego problem for certain analytic functions defined by ... 633

and

$$
a_{3}=\frac{p_{1} \omega_{2}}{[3]_{q}-1}+\frac{p_{2} \omega_{1}^{2}}{[3]_{q}-1}+\frac{p_{1}^{2} \omega_{1}^{2}}{\left([2]_{q}-1\right)\left([3]_{q}-1\right)} .
$$

Hence, by (2.5) and (2.6), we get the following

$$
a_{3}-\mu a_{2}^{2}=\frac{p_{1}}{[3]_{q}-1}\left(\omega_{2}+t \omega_{1}^{2}\right)
$$

where

$$
t=\frac{p_{2}}{p_{1}}-\frac{\mu p_{1}\left([3]_{q}-1\right)}{[2]_{q}^{2}} .
$$

The result (2.7) now follows from (1.17).

Following the proof of Theorem 2.1 and definitions 1.2, 1.3 and 1.4, we have

Theorem 2.2. Let $k \in[0, \infty), q \in(0,1)$ and let $p_{k}(z)=1+p_{1} z+p_{2} z^{2}+\ldots$ be defined as in the Definition $A$. If $f$ given by (1.1) belongs to $S_{c}^{*}\left(p_{k}\right)$ then we have

(2.9) $\left|a_{3}-\mu a_{2}^{2}\right| \leq \frac{p_{1}}{[3]_{q}-1} \max \left\{1,\left|\frac{p_{2}}{p_{1}}+\frac{p_{1}\left([2]_{q}-1\right)-\mu p_{1}\left([3]_{q}-1\right)}{\left([2]_{q}-1\right)^{2}}\right|\right\}$

(2.9) holds for any complex number $\mu$.

Theorem 2.3. Let $k \in[0, \infty), q \in(0,1)$ and let $p_{k}(z)=1+p_{1} z+p_{2} z^{2}+\ldots$ be defined as in the Definition $A$. If $f$ given by (1.1) belongs to $S_{s}^{c}\left(p_{k}\right)$ then we have

$$
\left|a_{3}-\mu a_{2}^{2}\right| \leq \frac{p_{1}}{3[3]_{q}-2} \max \left\{1,\left|\frac{p_{2}}{p_{1}}-\frac{3 \mu p_{1}\left([3]_{q}-2\right)}{4[2]_{q}^{2}}\right|\right\}
$$

(2.10) holds for any complex number $\mu$.

Theorem 2.4. Let $k \in[0, \infty), q \in(0,1)$ and let $p_{k}(z)=1+p_{1} z+p_{2} z^{2}+\ldots$ be defined as in the Definition $A$. If $f$ given by (1.1) belongs to $S_{c}^{c}\left(p_{k}\right)$ then we have

$$
\left|a_{3}-\mu a_{2}^{2}\right| \leq \frac{p_{1}}{3\left([3]_{q}-1\right)} \max \left\{1,\left|\frac{p_{2}}{p_{1}}+\frac{4\left([2]_{q}-1\right) p_{1}-3 \mu p_{1}\left([3]_{q}-1\right)}{4\left([2]_{q}-1\right)^{2}}\right|\right\}
$$

(2.11) holds for any complex number $\mu$. 


\section{References}

[1] R.M. Ali, S.K. Lee, V. Ravichandran and S. Supramaniam, The Fekete-Szego coefficient functional for transforms of analytic functions, Bulletin of the Iranian Mathematical Society, 35 (2), pp. 119-142, (2009).

[2] A. Aral, V. Gupta and R.P. Agarwal, Applications of q-Calculus in Operator Theory, Springer-Verlag, New York, (2013).

[3] A.M. Gbolagade, S.O. Olatunji and T. Anake, Coefficient bounds for certain classes of analytic and univalent functions as related to sigmoid function, International Electronic Journal of Pure and Applied Mathematics, 7 (1), pp. 41-51, (2014).

[4] R.M. Goel and B.C. Mehrok, A subclass of starlike functions with respect to symmetric points, Tamkang Journal of Mathematics, 13 (1), pp. 11-24, (1982).

[5] S. Hussain, M.A. Alamri and M. Darus On a new class of $(j, i)$-symmetric function on conic regions, Journal of Nonlinear Sciences and Applications, 10, pp. 4628-4637, (2017).

[6] F. H. Jackson, On q-definite integrals, The Quarterly Journal of Pure and Applied Mathematics, 28, pp. 193-203, (1910).

[7] F. H. Jackson, On q-functions and a certain difference operator, Transactions of the Royal Society of Edinburgh, 46, pp. 253-281, (1908).

[8] J.M. Jahangiri, C. Ramachandran and S. Annamalai, Fekete-Szego problem for certain analytic functions defined by hypergeometric functions and Jacobi Polynomial, Journal of Fractional Calculus and Applications, 9 (1), pp. 1-7, (2018).

[9] S. Kanas and A. Wisniowska, Conic regions and k-uniform convexity, continued fractions and geometric function theory (CONFUN), Trondheim, (1997) Journal of Computational and Applied Mathematics, 105, pp. 327-336, (1999).

[10] S. Kanas and A. Wisniowska, Conic domains and starlike functions, Revue Roumaine des Mathematiques Pures et Appliquees, 45, pp. 645$658,(2000)$. 
[11] N. Magesh, S. Altinkaya and S. Yalcin, Construction of Toeplitz matrices whose elements are coefficient of univalent functions associated with q-derivative operator, arxiv: 1708.03600v1[Math.CV], (2017).

[12] N. Magesh, S. Altinkaya and S. Yalcin, Certain subclasses of $k$-uniformly starlike functions associated with symmetric $q$-derivative operator, Journal of Computational Analysis and Applications, 24 (8), pp. 1464-1473, (2018).

[13] S.D. Purohit and R.K. Raina, Fractional q-calculus and certain subclasses of univalent analytic functions, Mathematica, 55 (78) (1), pp. 62-74, (2013).

[14] C. Selvaraj and N. Vasanthi, Subclasses of analytic functions with respect to symmetric and conjugate points, Tamkang Journal of Mathematics, 13 (1), pp. 11-24, (1982).

\section{O. Olatuntji}

Department of Mathematical Sciences,

Federal University of Technology,

P. M. B. 704,

Akure,

Nigeria

e-mail : olatunjiso@futa.edu.ng

and

\section{H. Dutta}

Department of Mathematics, Gauhati University,

Guwahati-781014

India

hemen_dutta08@rediffmail.com 